Research Article

Genomics and Bioinformatics

\title{
Metabarcoding reveals that a non-nutritive sweetener and sucrose yield similar gut microbiota patterns in Wistar rats
}

Tiago Falcon ${ }^{1}$ (D), Kelly Carraro Foletto ${ }^{2}$, Marina Siebert ${ }^{3}$, Denise Entrudo Pinto ${ }^{2}$, Michael Andrades ${ }^{3}$ and Marcello Casaccia Bertoluci ${ }^{2,4}$

${ }^{1}$ Hospital de Clínicas de Porto Alegre (HCPA), Centro de Pesquisa Experimental, Núcleo de Bioinformática, Porto Alegre, RS, Brazil.

${ }^{2}$ Universidade Federal do Rio Grande do Sul (UFRGS), Programa de Pós-Graduação em Medicina: Ciências Médicas, Porto Alegre, RS, Brazil.

${ }^{3}$ Hospital de Clínicas de Porto Alegre (HCPA), Centro de Pesquisa Experimental, Unidade de Pesquisa

Laboratorial, Porto Alegre, RS, Brazil.

${ }^{4}$ Hospital de Clínicas de Porto Alegre (HCPA), Serviço de Endocrinologia, Porto Alegre, RS, Brazil.

\begin{abstract}
The effects of non-nutritive sweeteners (NNS) on the gut microbiota are an area of increasing research interest due to their potential influence on weight gain, insulin resistance, and inflammation. Studies have shown that mice and rats fed saccharin develop weight gain and metabolic alterations, possibly related to changes in gut microbiota. Here, we hypothesized that chronic exposure to a commercial NNS would change the gut microbiota composition in Wistar rats when compared to sucrose exposure. To test this hypothesis, Wistar rats were fed either NNS- or sucrose-supplemented yogurt for 17 weeks alongside standard chow (ad libitum). The gut microbiome was assessed by $16 S$ rDNA deep sequencing. Assembly and quantification were conducted using the Brazilian Microbiome Project pipeline for lon Torrent data with modifications. Statistical analyses were performed in the $\mathrm{R}$ software environment. We found that chronic feeding of a commercial NNS-sweetened yogurt to Wistar rats, within the recommended dose range, did not significantly modify gut microbiota composition in comparison to sucrose-sweetened yogurt. Our findings do not support the hypothesis that moderate exposure to NNS is associated with changes in gut microbiota pattern compared to sucrose, at least in this experimental model.
\end{abstract}

Keywords: $16 S$ rDNA, deep sequencing, saccharin, cyclamate, yogurt.

Received: January 30, 2019; Accepted: January 13, 2020.

\section{Introduction}

Non-nutritive sweeteners (NNS) impart a sweet taste to foodstuffs without adding caloric value, and are thus widely used in the management of overweight, glucose intolerance, and other metabolic diseases (Fitch et al., 2012). This class of molecules was first considered inert, but chronic consumption has raised concerns regarding user health and environmental safety (Pepino, 2015; Praveena et al., 2018). Experimental studies suggest that NNS are not as inert as initially thought; dopaminergic degeneration (Amin et al., 2018), changes in the gut microbiota (Suez et al., 2014; Bian et al., 2017a; Uebanso et al., 2017), and cell barrier disruption in vitro (Santos et al., 2018) have been described as consequences of their chronic intake.

Send correspondence to Tiago Falcon. Hospital de Clínicas de Porto Alegre (HCPA), Centro de Pesquisa Experimental, Núcleo de Bioinformática, Rua Ramiro Barcelos, 2350, CEP 90035-903, Porto Alegre, RS Brasil. Email: tiagofalconlopes@gmail.com
Epidemiological studies have shown that NNS intake is paradoxically associated with increased weight gain and adiposity, the metabolic syndrome, type 2 diabetes mellitus, and cardiovascular disease (Colditz et al., 1990; Dhingra et al., 2007; Fowler et al., 2008; Lutsey et al., 2008). In previous experiments, we found that rats fed an isocaloric diet supplemented with commercial NNS gained more weight than rats fed a sucrose-supplemented diet (Feijó et al., 2013; Pinto et al., 2017) or a non-sweetened diet (Foletto et al., 2016). Some mechanisms have been proposed to explain the metabolic alterations seen after NNS use, such as changes in energy expenditure rate (Pinto et al., 2017), interaction with sweet receptors (with consequences for insulin secretion), and changes in the gastrointestinal environment (Pepino, 2015). Emerging evidence has shown that gut microbiota composition is associated with metabolic diseases (Akbari and Hendijani, 2016; Boulangé et al., 2016). Furthermore, the balance between resident microorganisms may predispose the host to weight 
gain by facilitating recovery of nutrients from food (Turnbaugh et al., 2006). This phenomenon has been demonstrated by fecal transplantation from obese humans to lean mice, which eventually acquired the phenotype of the donors (Goodrich et al., 2014). Some studies have shown that the gut microbiome is disturbed by chronic exposure to several NNS, including acesulfame potassium, aspartame, cyclamate, neotame, saccharin, sucralose, and steviol glycosides (Lobach et al., 2018).

Although these studies are important, results still need to be confirmed for different NNS types, doses, and degrees of exposure. In the present study, we hypothesized that chronic intake of a commercially available NNS $(0.3 \%$ sodium saccharin and sodium cyclamate, Zero-Cal) would induce changes in the rat gut microbiome.

\section{Materials and Methods}

\section{Study design and animals}

The present study is a reanalysis of previous research; details are given elsewhere (Pinto et al., 2017). In brief, adult male Wistar rats weighing $210 \pm 6$ grams (mean \pm SE) were ranked according to the baseline weight and assigned to receive NNS-supplemented yogurt (NNS, $\mathrm{n}=10)$ or sucrose-sweetened yogurt (SUC, $n=9$ ) in" a block randomization approach.

All the animals received standard chow and water $a d$ libitum and were kept individually in translucent polypropylene cages with controlled humidity (65-70\%), temperature $\left(22 \pm 1^{\circ} \mathrm{C}\right)$, and light-dark cycle (12/12 hours).

After 17 weeks, the animals were euthanized by decapitation, the abdomen was opened, and the distal portion of the large intestine was removed. The fecal pellets were moved directly to a sterile cryotube which was immediately frozen in liquid nitrogen and stored in a freezer at $-80{ }^{\circ} \mathrm{C}$.

Trained researchers were blinded to group allocation. All the procedures were reviewed and approved by the local institutional animal care and use committee (Comissão de Ética no Uso de Animais em Pesquisa do Hospital de Clínicas de Porto Alegre, protocol number 16-0011) and conducted in accordance with Brazilian law. This manuscript was written in accordance with the ARRIVE guidelines (Kilkenny et al., 2010).

\section{Dietary manipulation}

All rats received standard chow pellets $(2.93 \mathrm{kcal} / \mathrm{g})$ (Nuvital CR-1, Nuvilab) ad libitum. Chow was added to the top of the cages every 24 hours as needed, and remaining chow was weighed and recorded once a week with an electronic precision scale (AS 5500, Marte, SP, Brazil). Intake was thus calculated and recorded weekly. The largest solid pellets were allocated in the grid feeders. A bottom crumb collector on the outside of the cage was installed to minimize losses. Cages were carefully monitored for any evi- dence of chow spillage and crumbs were considered for the control of chow intake.

Sweetened yogurt supplements were prepared according to an established protocol, described elsewhere (Feijó et al., 2013; Foletto et al., 2016; Pinto et al., 2017). Briefly, $20 \mathrm{~mL}$ of standardized low-fat yogurt (Nestlé, SP, Brazil) was supplemented with either $20 \%$ sucrose (União, SP, Brazil) or a commercial NNS $(0.3 \%$ sodium saccharin and sodium cyclamate, Zero-Cal, SP, Brazil). Additionally, $15 \mathrm{~mL}$ of pure water was added into yogurt to dilute and adjust viscosity to allow easier drinking, yielding a solution of $11.4 \%$ sucrose or $0.17 \%$ NNS. Yogurt supplements were offered for 22 hours each day (from 11 AM to 9 AM), 7 days a week, throughout the experiment. Yogurt was offered in special bottles with adapted nozzles to avoid leakage. The caloric densities of sucrose- and NNS-supplemented yogurt were $0.63 \mathrm{kcal} / \mathrm{mL}(\sim 170 \mathrm{kcal} / \mathrm{wk})$ and $0.24 \mathrm{kcal} / \mathrm{mL}(\sim 60 \mathrm{kcal} / \mathrm{wk})$, respectively. All rats ingested more than $65 \%$ of the yogurt supplement offered. The yogurt bottles were also checked for any sign of leakage or clogging. Water bottles were changed every 2 or 3 days and leftover water volumes were recorded. No preference for any beverage was noted during the study.

\section{DNA extraction and sequencing of the $16 S$ gene}

Bacterial DNA was extracted from fecal pellets using the QIAmp DNA Stool Mini Kit (Qiagen) and then quantitated using the Qubit dsDNA HS assay kit (Thermo Fisher Scientific) in a Qubit fluorimeter (Invitrogen).

Approximately $50 \mathrm{ng}$ of DNA was used for amplification of the V4 hypervariable region of the bacterial $16 \mathrm{~S}$ rRNA encoding gene by the polymerase chain reaction (PCR). The resulting product was purified and used in the preparation of the emulsion PCR followed by the sequencing reaction in an Ion Torrent Personal Genome Machine (PGM) System (Life Technologies). Sequencing data were processed in QIIME software. Bacterial diversity analyses were based on the degree of similarity between $16 \mathrm{~S}$ rDNA sequences, which were grouped into Operational Taxonomic Units (OTUs).

\section{Sequencing data analysis}

We followed the Brazilian Microbiome Project (BMP) $16 \mathrm{~S}$ profiling analysis pipeline for Ion Torrent (Pylro et al., 2014) with a few modifications. The BMP pipeline uses a mix of the UPARSE (Edgar, 2013) and QIIME (v 1.9.1) (Caporaso et al., 2010) pipelines. UPARSE is used for chimera removal and de novo OTU picking and quantification, while QIIME is used to map and classify the OTUs based on the Greengenes database (13_8), generate the output table, and perform alpha and beta diversity analyses. Using QIIME ${ }^{\mathrm{TM}}$, we changed the standard classifier from uclust to rdp. The rdp classifier (Wang et al., 2007) in QIIME ${ }^{\mathrm{TM}}$ uses the Greengenes database sequences to train the classifier and thus perform OTU 
classification. Another difference was that the BMP pipeline suggests the QIIME ${ }^{\mathrm{TM}}$ default of 0.5 as the least confidence to record an assignment; here, we used a least confidence of 0.8 .

\section{Statistical analysis}

All data were normalized using Aitchison's log-ratio (Aitchison, 1982, 1986), as we are working with compositional data (Gloor et al., 2017). The R software (v 3.4.4) (R Core Team, 2018), package vegan (v. 2.4) (Oksanen et al., 2017), was used to perform the diversity analyses (alpha and beta diversities, Shannon and Simpson diversity indices, and richness comparisons) and their respective plots. The Shapiro-Wilk test of normality was applied to all identified taxa levels, except those unclassified (Table S1). Then, Student's $t$-test or the Wilcoxon rank-sum test were used in accordance with the value distribution ( $t$-test for parametric and Wilcoxon for non-parametric data). All p-values were corrected using the false discovery rate (FDR) method. Only those taxa comparisons with FDR < 0.05 were considered as a statistically significant difference between groups. Unclassified taxa were used only for the diversity analyses.

\section{Results}

Yogurt intake was similar in the NNS- and SUCsupplemented groups over the 17-week study period. NNS intake in the NNS group was stable over time (median = $154 \mu \mathrm{L} / \mathrm{kg} /$ day; IQR $=39 \mu \mathrm{L} / \mathrm{kg} /$ day) (Figure $\mathrm{S} 1$ ).

Diversity analyses indicated no significant difference between the NNS or SUC groups regarding species richness (Figure 1a), or Shannon (ranging from 3.410 to 4.574) or Simpson (ranging from 0.891 to 0.975 ) (File S1) diversity indices $(\mathrm{p}=0.297$ and $\mathrm{t}=-1.085, \mathrm{p}=0.551$ and $\mathrm{t}=$ $0.611, p=0.198$ and $t=1.356$, respectively). Samples show overlapping rarefaction curves and a similar number of species per read counts per sample (Figure 1b). The weighted beta diversity approximates the centroids, mixing samples from both groups (Figure 1c). Proportion data for all identified taxa, analyzed one by one at all taxonomic levels, were similar in the NNS and SUC groups, with no significant differences (Figure 2, File S2).

\section{Discussion}

Concerns about chronic intake of NNS have been raised in recent years. However, here we present evidence that addition of NNS- or SUC-sweetened yogurt to a standard chow diet for 17 weeks yielded similar microbiota composition and high diversity indices.

We previously described that Wistar rats fed yogurt supplemented with a daily recommended dose of commercial NNS for 17 weeks gained more weight than animals fed sucrose-supplemented yogurt. This phenomenon could be partially explained by a decrease in energy expenditure a

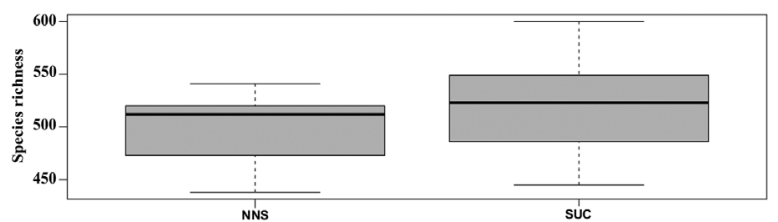

b

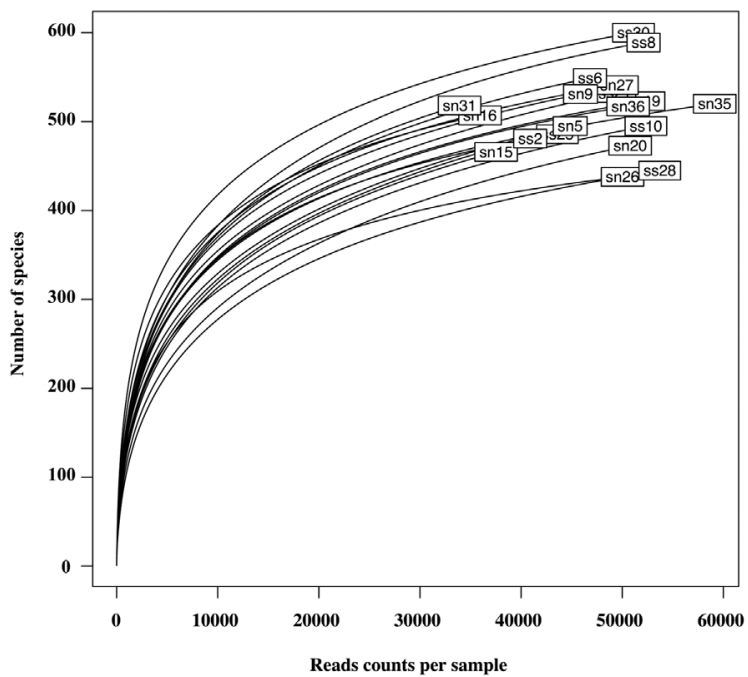

C

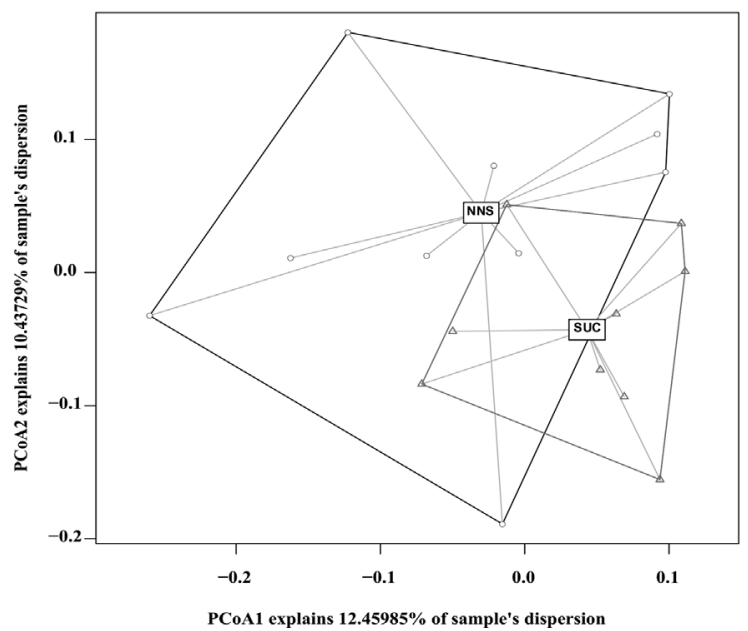

Figure 1 - Diversity analysis plots. (a) Species richness boxplot. The dark line inside the boxplots is the median, separating the upper quantile. Error bars represent the standard deviation. (b) Rarefaction plot per sample. sn, samples belonging to the NNS group; ss, samples belonging to the SUC group. Numbers indicate the number of the rat in the box for internal annotation. (c) Weighted beta diversity plot using principal coordinate analysis. Black circles represent the NNS samples; red triangles represent the SUC samples. Dark lines connect the most distant samples from the centroid of the NNS group, while red lines connect the most distant samples from the centroid of the SUC group. NNS, non-nutritive sweetener $(\mathrm{n}=$ $10)$; SUC, sucrose $(\mathrm{n}=9)$.

at rest (Pinto et al., 2017). Our findings are in line with epidemiological studies that have associated NNS with weight gain, the metabolic syndrome, type 2 diabetes mellitus, and cardiovascular disease (Colditz et al., 1990; Dhingra et al., 2007; Fowler et al., 2008; Lutsey et al., 2008). Other mechanisms have also been proposed to explain this counterintuitive effect, such as NNS interaction with sweet receptors followed by changes in glucose absorption and insulin 
secretion and changes in the gastrointestinal environment (Pepino, 2015).

Because of the intimate relationship between host microbiota and nutritional availability, this mechanism has a

$$
\begin{array}{r}
\text { Bacteria } \\
\square \text { Archaea }
\end{array}
$$

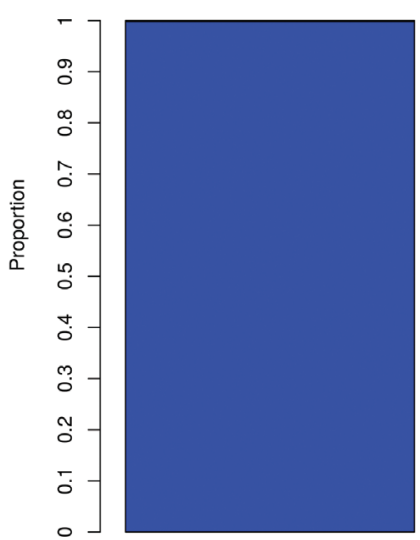

c

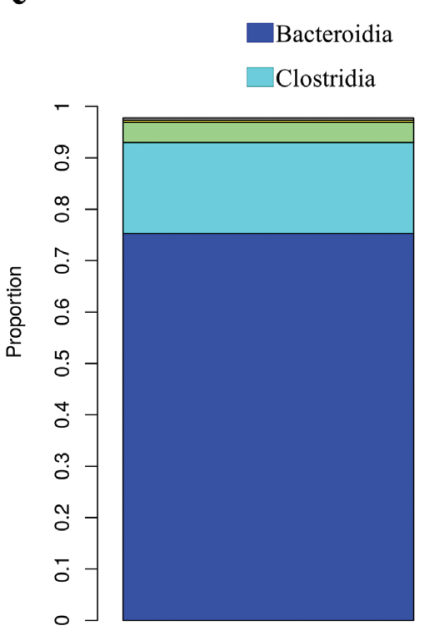

e

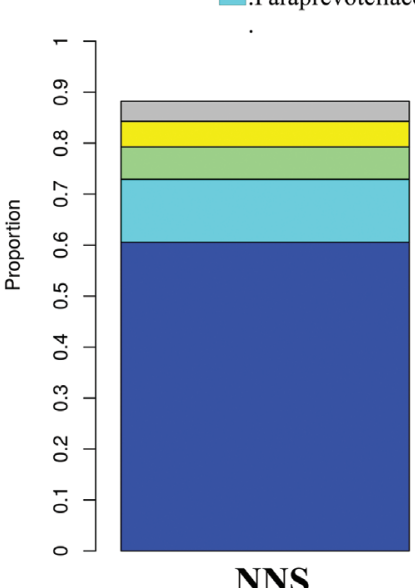

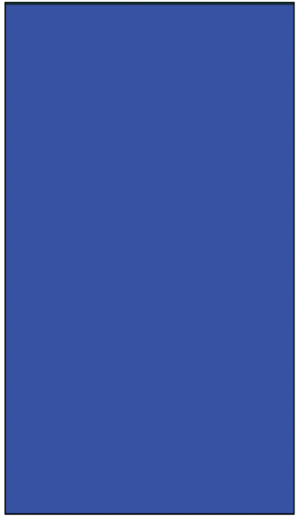

\section{Bacili}

$\square$ Betaproteobacteria

$\square$ 4C0d.2

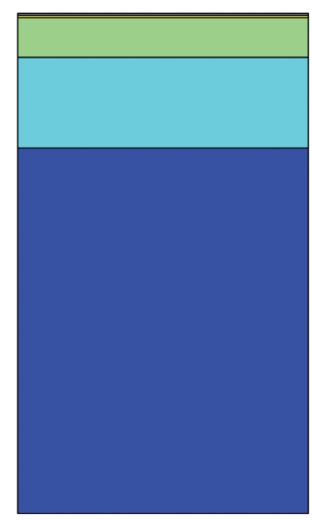

Ruminococcaceae S24.7

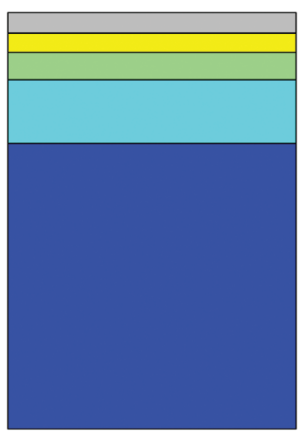

SUC

become widely explored. In fact, evidence points to a harmful effect of NNS on the microbiome, with acesulfame potassium, aspartame, cyclamate, neotame, saccharin, sucralose, and steviol glycosides all potentially associated

b

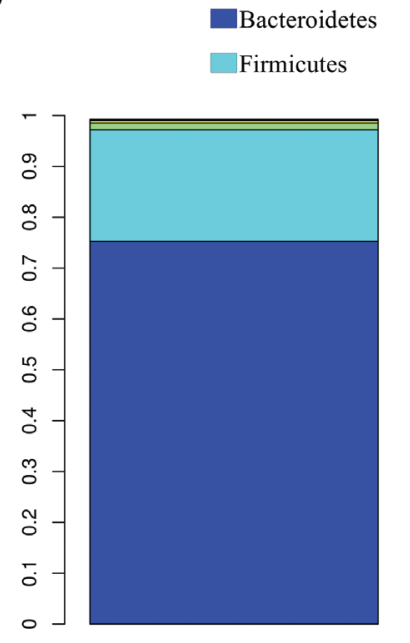

d
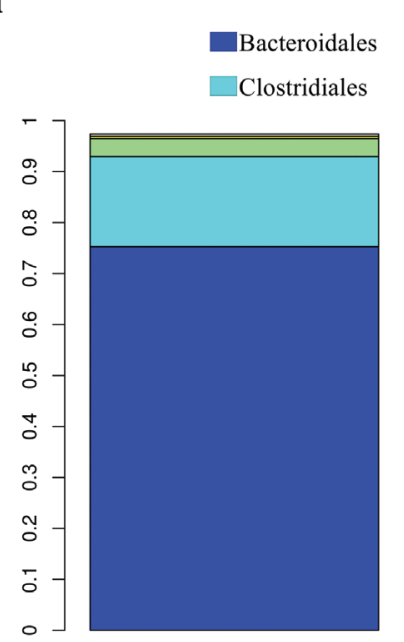

f

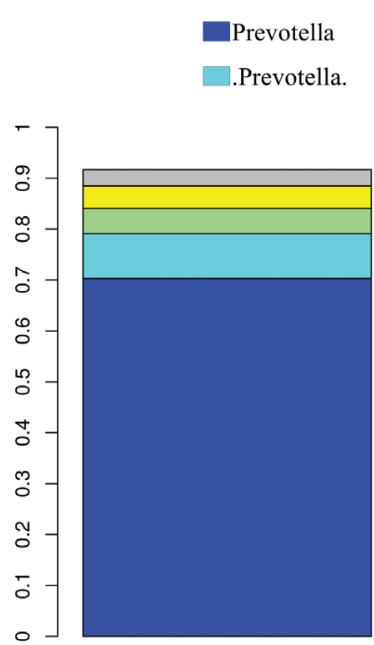

NNS $\square$ Proteobacteria

Cyanobacteria

$\square$ Spirochaetes

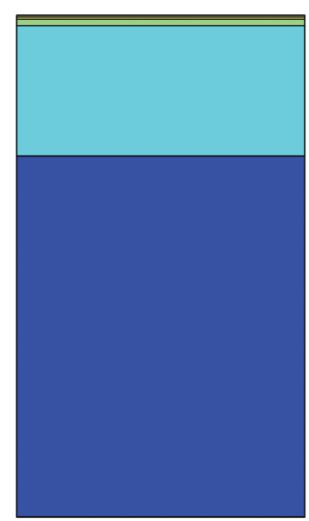

Lactobacillales Burkholderiales

$\square$ YS2

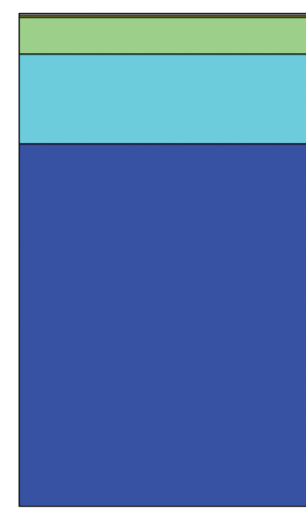

$\mathrm{CF} 231$

Lactobacillus

$\square$ Oscillospira

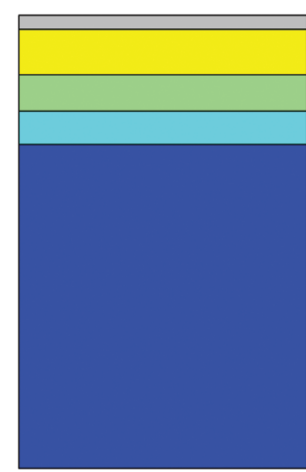

SUC

Figure 2 - Average proportion of the two represented (a) domains and the five most representative (b) phyla, (c) classes, (d) orders, (e) families, and (f) genera among the experimental groups. Color key at the top of each figure. NNS, non-nutritive sweetener $(n=10)$; SUC, sucrose $(n=9)$. 
with dysbiosis (Lobach et al., 2018). Regarding saccharin use, Suez et al., 2014 described 40 altered OTUs and glucose intolerance in mice receiving high doses in tap water for 11 weeks.

Despite these results, in the present study, we found that, when added to the diet at a level equivalent to recommended human adult doses, a commercial NNS widely employed as a food additive had no effect on the rat microbiota, even with chronic consumption. Our results run counter to those of previous studies that offered saccharin at doses far beyond the acceptable human daily intake (ADI) to the animals (Anderson and Kirkland, 1980; Suez et al., 2014). Similar results have been found for other NNS: acesulfame potassium and aspartame, given at 2.5 times or 30 times the ADI, respectively, promoted alterations in the gut microbiota (Suez et al., 2014; Bian et al., 2017a), whereas no such alterations were found when the ADI was respected (Palmnäs et al., 2014; Uebanso et al., 2017). One exception is the gut dysbiosis and liver inflammatory markers seen in mice receiving water supplemented with saccharin at an ADI-equivalent dose $(0.3 \mathrm{mg} / \mathrm{mL})$ (Bian et al., 2017b). In this line, Lobach et al. (2018) recently raised concerns about the real cause of dysbiosis caused by NNS.

Besides using a quantity of NNS equivalent to usual human exposure, we also mixed the sweetener with a food product. This is a noteworthy difference from previous studies, in which NNS were usually given dissolved in the tap water. We are aware that yogurts contain live microorganisms, and their consumption can alter the gut microbial community (Lisko et al., 2017). We recognize this may be perceived as a limitation of the present study. However, we used a randomized controlled design in which both groups (NNS and SUC) received yogurt, normalizing the potential impact of its components on the gut microbiota. Thus, we understand that the use of yogurt in the present study represents a strength rather than a limitation, as in real-world circumstances, NNS are usually consumed mixed with food and beverages and not in pure water.

Our findings also suggest three other hypotheses that should be tested in future: (i) gut microbiota composition is not altered by saccharin, independently of the sweetener carrier. Unfortunately, most previous studies have tested saccharin in water at doses exceeding the ADI range, which hinders comparisons. One recent study showed changes in gut microbiome and in lung inflammatory markers after 6 months of exposure to saccharin within human ADI range $(0.3 \mathrm{mg} / \mathrm{mL})$ (Bian et al., 2017b). Still, a well-designed experiment comparing water to food carriers is necessary; (ii) yogurt may act as the main modifying agent of the gut microbiota; or (iii) yogurt may act to maintain microbiota homeostasis despite NNS exposure.

\section{Conclusion}

Our hypothesis of an association between chronic intake of a commercially available NNS and changes in gut microbiota was refuted. Rats on a prolonged diet of exposure to sucrose or moderate doses of NNS diluted in low-fat yogurt showed a similar microbiota composition pattern.

\section{Data Access}

Raw sequence data and information about the barcodes were submitted to the NCBI BioProject database under the number PRJNA480648.

\section{Acknowledgments}

We thank the reviewer for his/her valuable comments.

T.F. received funding support through a fellowship from Programa Nacional de Pós-Doutorado (PNPD) CAPES/HCPA (process no.: 88887.160608/2017-00). This study received financial support from FIPE/HCPA. This study was financed in part by the Coordenação de Aperfeiçoamento de Pessoal de Nível Superior - Brasil (CAPES) - Finance Code 001.

\section{Conflict of Interest}

The authors declare that there is no conflict of interest that could be perceived as prejudicial to the impartiality of the reported research.

\section{Authors Contributions}

$\mathrm{TF}$ analyzed the data and wrote the manuscript; $\mathrm{KCF}$, MS and DEP conducted the experiments; MA and MCB conceived the study and wrote the manuscript. All authors read and approved the final version

\section{References}

Aitchison J (1982) The statistical analysis of compositional data. J R Stat Soc Series B Stat Methodol 44:139-177.

Aitchison J (1986) The statistical analysis of compositional data. Chapman and Hall, London.

Akbari V and Hendijani F (2016) Effects of probiotic supplementation in patients with type 2 diabetes: systematic review and meta-analysis. Nutr Rev 74:774-784.

Amin SN, Hassan SS and Rashed LA (2018) Effects of chronic aspartame consumption on MPTP-induced Parkinsonism in male and female mice. Arch Physiol Biochem 124:292-299.

Anderson RL and Kirkland JJ (1980) The effect of sodium saccharin in the diet on caecal microflora. Food Cosmet Toxicol 18:353-355.

Bian X, Chi L, Gao B, Tu P, Ru H and Lu K (2017a) The artificial sweetener acesulfame potassium affects the gut microbiome and body weight gain in CD-1 mice. PLoS One 12:e0178426.

Bian X, Tu P, Chi L, Gao B, Ru H and Lu K (2017b) Saccharin induced liver inflammation in mice by altering the gut micro- 
biota and its metabolic functions. Food Chem Toxicol 107:530-539.

Boulangé CL, Neves AL, Chilloux J, Nicholson JK and Dumas ME (2016) Impact of the gut microbiota on inflammation, obesity, and metabolic disease. Genome Med 8:42.

Caporaso JG, Kuczynski J, Stombaugh J, Bittinger K, Bushman FD, Costello EK, Fierer N, Peña AG, Goodrich JK, Gordon JI et al. (2010) QIIME allows analysis of high-throughput community sequencing data. Nat Methods 7:335-336.

Colditz GA, Willett WC, Stampfer MJ, London SJ, Segal MR and Speizer FE (1990) Patterns of weight change and their relation to diet in a cohort of healthy women. Am J Clin Nutr 51:1100-1105.

Dhingra R, Sullivan L, Jacques PF, Wang TJ, Fox CS, Meigs JB, D'Agostino RB, Gaziano JM and Vasan RS (2007) Soft drink consumption and risk of developing cardiometabolic risk factors and the metabolic syndrome in middle-aged adults in the community. Circulation 116:480-488.

Edgar RC (2013) UPARSE: highly accurate OTU sequences from microbial amplicon reads. Nat Methods 10:996-998.

Feijó FM, Ballard CR, Foletto KC, Batista BAM, Neves AM, Ribeiro MFM and Bertoluci MC (2013) Saccharin and aspartame, compared with sucrose, induce greater weight gain in adult Wistar rats, at similar total caloric intake levels. Appetite 60:203-207.

Fitch C, Keim KS and Academy of Nutrition and Dietetics (2012) Position of the Academy of Nutrition and Dietetics: use of nutritive and nonnutritive sweeteners. J Acad Nutr Diet 112:739-758.

Foletto KC, Batista BAM, Neves AM, Feijó FM, Ballard CR, Ribeiro MFM and Bertoluci MC (2016) Sweet taste of saccharin induces weight gain without increasing caloric intake, not related to insulin-resistance in Wistar rats. Appetite 96:604-610.

Fowler SP, Williams K, Resendez RG, Hunt KJ, Hazuda HP and Stern MP (2008) Fueling the obesity epidemic? Artificially sweetened beverage use and long-term weight gain. Obesity 16:1894-1900.

Gloor GB, Macklaim JM, Pawlowsky-Glahn V and Egozcue JJ (2017) Microbiome Datasets Are Compositional: And This Is Not Optional. Front Microbiol 8:2224.

Goodrich JK, Waters JL, Poole AC, Sutter JL, Koren O, Blekhman R, Beaumont M, Van Treuren W, Knight R, Bell JT et al. (2014) Human genetics shape the gut microbiome. Cell 159:789-799.

Kilkenny C, Browne WJ, Cuthill IC, Emerson M and Altman DG (2010) Improving bioscience research reporting: the ARRIVE guidelines for reporting animal research. PLoS Biol 8:e1000412.

Lisko DJ, Johnston GP and Johnston CG (2017) Effects of Dietary Yogurt on the Healthy Human Gastrointestinal (GI) Microbiome. Microorganisms 15:E6.

Lobach AR, Roberts A and Rowland IR (2018) Assessing the in vivo data on low/no-calorie sweeteners and the gut microbiota. Food Chem Toxicol 124:385-399.

Lutsey PL, Steffen LM and Stevens J (2008) Dietary intake and the development of the metabolic syndrome: the Atherosclerosis Risk in Communities study. Circulation 117:754-761.

Palmnäs MSA, Cowan TE, Bomhof MR, Su J, Reimer RA, Vogel HJ, Hittel DS and Shearer J (2014) Low-dose aspartame consumption differentially affects gut microbiota-host met- abolic interactions in the diet-induced obese rat. PLoS One 9:e109841.

Pepino MY (2015) Metabolic effects of non-nutritive sweeteners. Physiol Behav 152:450-455.

Pinto DE, Foletto KC, Nunes RB, Lago PD and Bertoluci MC (2017) Long-term intake of saccharin decreases post-absortive energy expenditure at rest and is associated to greater weight gain relative to sucrose in wistar rats. Nutr Metab 14:18.

Praveena SM, Cheema MS and Guo HR (2018) Non-nutritive artificial sweeteners as an emerging contaminant in environment: A global review and risks perspectives. Ecotoxicol Environ Saf 170:699-707.

Pylro VS, Roesch LFW, Morais DK, Clark IM, Hirsch PR and Tótola MR (2014) Data analysis for $16 \mathrm{~S}$ microbial profiling from different benchtop sequencing platforms. J Microbiol Methods 107:30-37.

Santos PS, Caria CRP, Gotardo EMF, Ribeiro ML, Pedrazzoli J and Gambero A (2018) Artificial sweetener saccharin disrupts intestinal epithelial cells' barrier function in vitro. Food Funct 9:3815-3822.

Suez J, Korem T, Zeevi D, Zilberman-Schapira G, Thaiss CA, Maza O, Israeli D, Zmora N, Gilad S, Weinberger A et al. (2014) Artificial sweeteners induce glucose intolerance by altering the gut microbiota. Nature 514:181-186.

Turnbaugh PJ, Ley RE, Mahowald MA, Magrini V, Mardis ER and Gordon JI (2006) An obesity-associated gut microbiome with increased capacity for energy harvest. Nature 444:1027-1031.

Uebanso T, Ohnishi A, Kitayama R, Yoshimoto A, Nakahashi M, Shimohata T, Mawatari K and Takahashi A (2017) Effects of low-dose non-caloric sweetener consumption on gut microbiota in mice. Nutrients 9:560.

Wang Q, Garrity GM, Tiedje JM and Cole JR (2007) Naive Bayesian classifier for rapid assignment of rRNA sequences into the new bacterial taxonomy. Appl Environ Microbiol 73:5261-5267.

\section{Internet Resources}

Oksanen J, Blanchet FG, Friendly M, Kindt R, Legendre P, McGlinn D, Minchin PR, O'Hara RB, Simpson GL, Solymos P et al. (2017) Vegan: Community Ecology Package, https:/cran.r-project.org/web/packages/vegan/index.html. R Core Team (2018) R: a language and environment for statistical computing, https://www.gbif.org/pt/tool/81287/r-a-language-and-envi ronment-for-statistical-computing.

\section{Supplementary Material}

The following online material is available for this article:

Figure S1 - Yogurt (a) and NNS intake (b) over time.

File S1: Shannon and Simpson indices of diversity. The name of each sheet indicates the respective index.

File S2: One-by-one comparison of identified taxa (from phylum to genus).

Table S1 - Operational Taxonomic Unit (OTU) table.

\section{Associate Editor: Guilherme Correa de Oliveira}

License information: This is an open-access article distributed under the terms of the Creative Commons Attribution License (type CC-BY), which permits unrestricted use, distribution and reproduction in any medium, provided the original article is properly cited. 